\title{
Endogenous timing in a vertically differentiated mixed duopoly with Cournot competition
}

\author{
LEIDONG FENG and MENGDI GU
}

\begin{abstract}
This paper compares the equilibrium outcomes under simultaneous and sequential output setting in a mixed duopoly in a vertically differentiated market. When the timing of the output game is determined endogenously, it is shown that simultaneous play in the quality stage with the public firm acting as the high-quality producer and simultaneous play in the second period in the output stage turn out to be the unique subgame perfect Nash equilibrium, which contrasts with the endogenous timing in a purely private duopoly.
\end{abstract}

Key words: vertical differentiation, endogenous timing, public firm, private firm.

\section{Introduction}

Mixed oligopolies are observed commonly in most countries, in which state-owned public firms compete against private firms. In some industries, homogeneous goods (Pal [12] and $\mathrm{Lu}$ [9]) and horizontally differentiated products (such as Cremer et al. [3], Ba'rcena-Ruiz [1], and Ishibashi and Kaneko [7]) are provided. However, using the model of vertical product differentiation, the results may be different. In mixed markets, public and private firms compete not only in quality of their products, but in price or output. Although studies on mixed markets are growing in recent years, Cournot competition in mixed oligopoly has received few interests in the literature. Under the assumptions of fully covered markets and variable quality-dependent costs, Grilo [5] studied duopolistic mixed competition when products were vertically differentiated and firms chose simultaneously first qualities and then prices. The author proved that the socially optimal solution could be sustained as a market outcome by using a public firm as a market agent. Lutz and Pezzino [10] examined two standard market competition modes (Bertrand and Cournot) in a mixed market with a public firm and a private firm where both firms chose their prices in stage 1 and quantities in stage 2 simultaneously. They

L. Feng, the corresponding author is with Jiaxing University, School of Business, Jiaxing, China, email: ldfeng@ 126.com. M. GU is with Shanghai Jiao Tong University, Antai College of Economics and Management, Shanghai, China.

This work was supported by the Key Program of National Natural Science Foundation of China (No. 71132006).

Received 18.06.2015. 
found that mixed oligopoly was in general socially desirable compared with a private duopoly regardless of the type of competition in the short run and the equilibrium quality ranking.

The purpose of this paper is to addresses the issue of endogenous order of moves in a mixed oligopoly by adopting the observable delay game of Hamilton and Slutsky [6] in the context of output setting mixed oligopoly where the firms choose their timing of outputs simultaneously after the quality of their products are determined. Instead of exogenously specifying a simultaneous or a sequential move game, the order of move is endogenously determined from the model. It is shown that the results are strikingly different from those obtained in a corresponding output setting oligopoly with all profit maximizing firms.

To our knowledge, the early paper that considered sequential price setting in a mixed oligopoly using a vertical differentiated model is Delbono et al. [4]. They explicitly analyzed the endogenous quality choice in mixed oligopoly. Using the model of vertical differentiation in which the market is segmented by the high and low quality products, Delbono et al. found that there exists an equilibrium where the public firm chooses lower quality than the private firm. However, in their model, the other equilibrium where the public firm chooses higher quality also exists, and the latter equilibrium is more robust with respect to the timing of the game. Liu and $\mathrm{Lu}$ [8] considered a game of endogenous timing with observable delay in a mixed duopoly with endogenous vertical differentiation in the context of sequential quality and price choice. They found that a simultaneous play in the first period at each stage turns out to be the unique subgame perfect Nash equilibrium.

Therefore, the existing literature on mixed oligopoly by using vertical differentiated model have failed to pay attention to the outputs setting with Cournot competition. In this paper, we provide a model of mixed duopoly which is consistent with Cournot competition. Suppose that in stage one, the public and private firm choose quality simultaneously and their choice between high and low quality is endogenous. The public firm maximizes social welfare while the private firm its own profit. In stage two (the pre-play stage), after observing each firm's quality level, firms simultaneously announce in which period they will choose their outputs and are committed to this announcement. Finally in stage three, firms choose their outputs knowing when the other firm chooses its output level.

Our main findings are the following: First, when the timing of the output game is determined endogenously, it is shown that simultaneous play in the quality stage with the public firm acting as the high-quality producer and simultaneous play in the second period in the output stage turn out to be the unique subgame perfect Nash equilibrium of the game with observable delay, which contrasts with the endogenous timing in a purely private duopoly. Next, we show that the subgame perfect Nash equilibrium of the game under Cournot competition differs from Liu and $\mathrm{Lu}$ [8] under Bertrand competition.

The rest of the paper is organized as follows. Section 2 presents the model. The equilibriums of the output subgame and quality subgame are described in Sect. 3. In Sect. 
4, we compare with endogenous timing in the same setting in a purely private duopoly. Section 5 provides concluding remarks, and some proofs are given in the Appendix.

\section{The model}

We consider a model of a duopoly for vertical differentiated products. On the supply side, as in Motta [11], inter alia, the convex fixed costs of firms' quality improvement are specified as

$$
C_{i}\left(s_{i}, x_{i}\right)=\frac{1}{2} s_{i}^{2} x_{i}, \quad i=H, L
$$

where $s_{i}$ and $x_{i}$ denote the quality and output produced by firm $i$, and $s_{H}>s_{L}>0$. Following the concept given in Choi and Shin [2], there is a continuum of consumers whose types are identified by $\theta$, uniformly distributed with density equal to 1 in the interval $[\underline{v}, \bar{v}]$, with $\bar{v}=\underline{v}+1$. Parameter $\theta$ denotes the consumers' marginal willingness to pay for quality. Each consumer has unit demand for the vertical differentiated product and the utility function:

$$
U= \begin{cases}\theta s_{i}-p_{i} & \text { if they buy one unit of the product with quality } s_{i} \text { at price } p_{i} \\ 0 & \text { otherwise. }\end{cases}
$$

This utility function is the one as described in Tirole [13]. Let $\hat{\theta}$ and $\widetilde{\theta}$ denote the marginal willingness to pay for quality identifying, respectively, the consumer who is indifferent between buying either the high quality or the low quality product and the one who is indifferent between buying the low quality product and not to buy at all

$$
\begin{gathered}
\hat{\theta}=\frac{p_{H}-p_{L}}{s_{H}-s_{L}} \\
\widetilde{\theta}=\frac{p_{L}}{s_{L}} .
\end{gathered}
$$

In this paper, our analysis considers the partial market coverage. The demand for the high and low quality products are given by $x_{H}=\bar{v}-\hat{\theta}$ and $x_{L}=\hat{\theta}-\widetilde{\theta}$ respectively. This is what one need to use in order to model Bertrand behavior, while inverse demands

$$
\begin{gathered}
p_{H}=s_{H}\left(\bar{v}-x_{H}\right)-s_{L} x_{L} \\
p_{L}=\left(\bar{v}-x_{H}-x_{L}\right) s_{L}
\end{gathered}
$$

are to be used under Cournot competition.

The public firm aims at maximizing social welfare and the objective function is

$$
W=\int_{\bar{v}-x_{H}}^{\bar{v}} \theta s_{H} \mathrm{~d} \theta+\int_{\bar{v}-x_{H}-x_{L}}^{\bar{v}-x_{H}} \theta s_{L} \mathrm{~d} \theta-\frac{1}{2} s_{H}^{2} x_{H}-\frac{1}{2} s_{L}^{2} x_{L}
$$


and the private firm's objective function is

$$
\pi_{i}= \begin{cases}\left(s_{H}\left(\bar{v}-x_{H}\right)-s_{L} x_{L}-\frac{1}{2} s_{H}^{2}\right) x_{H} & \text { if } i=H \\ \left(\left(\bar{v}-x_{H}-x_{L}\right) s_{L}-\frac{1}{2} s_{L}^{2}\right) x_{L} & \text { if } i=L .\end{cases}
$$

We consider a three-stage game as described in the Introduction. In the first stage, although the timing of choosing quality is given to be simultaneous exogenously, both firms can determine the quality of their products endogenously. They announce their time of setting their outputs simultaneously in stage two and last choose their outputs in the output stage. A major point of our model is that the choice of timing in the output game is made after the quality of two firms' products are determined. Our objective is to solve for the subgame perfect Nash equilibrium (or equilibrium) of this extend game with observable delay using backward induction.

\section{Results}

\subsection{Endogenizing the timing in the output stage}

In this paper, the basic output game is played in two periods, $F$ and $S$. In a prestage of timing choices, two firms simultaneously announce a period in which it will set the output of its product. Once selected, they are committed to it and their timing choices are public information. The basic output game is then played accordingly. If both firms select the same period in the prestage, $F$ or $S$, they play simultaneously in the basic game and the Cournot-Nash equilibrium results. If one firm selects $F$ and the other $S$, then a sequential play equilibrium results and the firm selecting $F$ acts as the leader.

Using backward induction, we obtain each firm's payoff in fixed-timing games, namely, the simultaneous-move, public-leader, and public-follower games. After that, we can then obtain the endogenous timing in the output stage. Since we do not know which firm produces higher quality product, we need to distinguish two cases, namely the case of the public firm providing lower quality product and the case of the public firm providing higher quality product. As mentioned in the introduction, which firm produces high quality product is endogenously determined. A detailed analysis is provided in the Appendix A.

\section{Proposition 1}

1. When $\frac{s_{H}}{s_{L}} \geqslant \frac{9}{8}$, both the public and private firm setting outputs in the second period is the unique strictly dominant strategy equilibrium in the output stage no matter which firm provides higher quality product.

2. When $1<\frac{s_{H}}{s_{L}}<\frac{9}{8}$, the unique strictly dominant strategy equilibrium in the output stage is sequential output settings with the private firm acting as the output leader no matter which firm provides higher quality product. 
Proof Let the superscripts ' $S$ ', ' $L$ ' and ' $F$ ' denote 'simultaneous-mover', 'leader' and 'follower', respectively.

1. In the case of the public firm providing lower-quality product, we present each firm's payoff in fixed-timing games in the Appendix A and have the following conclusion. When $\frac{s_{H}}{s_{L}} \geqslant \frac{9}{8}$, clearly, we have $W_{L}^{L}>W_{L}^{S}$ and $\pi_{H}^{L}>\pi_{H}^{S}$ since a leader can always choose its simultaneous-move equilibrium output while the leader actually chooses a different output. These inequalities mean that a firm wants to acts as a leader if the other firm moves in the first period. We also find $W_{F}^{L}>W_{L}^{L}$ and $\pi_{H}^{F}>\pi_{H}^{L}$. These inequalities signify that a firm wants to acts as a follower if the other firm moves in the second period. Nevertheless, when $1<\frac{s_{H}}{s_{L}}<\frac{9}{8}$, we have the different results $W_{L}^{F}>W_{L}^{L}>W_{L}^{S}$ and $\pi_{L}^{L}>\pi_{L}^{F}>\pi_{L}^{S}$.

2. In the case of the public firm providing higher quality product, we present each firm's payoff in fixed-timing games and derive the same conclusions in the Appendix A.

The proposition 1 signifies that two firms' action about output is determined by the level of differentiation between high quality and low quality after the quality stage. The result is different from the one in Lin and $\mathrm{Lu}$ [8] who investigated endogenous timing in a vertical differentiated mixed duopoly when firms compete in price, and the product differentiation in their model is also endogenous. Lin and $\mathrm{Lu} \mathrm{[8]} \mathrm{demonstrated} \mathrm{that} \mathrm{both}$ the public and private firm setting prices in the first period is the unique strictly dominant strategy equilibrium in the price stage no matter which firm provides higher quality product.

\subsection{Endogenizing the quality in the quality stage}

Having found the unique Nash equilibrium in the output stage, we can now investigate the endogenous quality. Since we exogenously assume that two firms set quality simultaneously, the key point is their choice of quality. We can get the following proposition.

\section{Proposition 2}

1. In the quality stage, the differentiation of equilibrium quality of products between the public and private firm is not less than $\frac{9}{8}$.

2. The unique strictly dominant strategy equilibrium in the quality stage is that the public firm acts as the higher quality producer.

\section{Proof}

1. A detailed analysis is provided in the Appendix B. Clearly, when $\frac{s_{H}}{s_{L}}<\frac{9}{8}$, we can identify that the equilibrium quality under this case, that is, two firms choose outputs sequentially with the private firm acting as the output leader no matter which firm provides higher quality product, contradict with the condition $\frac{s_{H}}{s_{L}}<\frac{9}{8}$ by using 
the reduction to absurdity. However, when $\frac{s_{H}}{s_{L}} \geqslant \frac{9}{8}$, two firms' equilibrium quality are

$$
\left(s_{H}, s_{L}\right)=(0.913549 \bar{v}, 0.588302 \bar{v})
$$

and

$$
\left(s_{H}, s_{L}\right)=(0.731408 \bar{v}, 0.320684 \bar{v})
$$

under different quality choice, respectively, which satisfy the condition $\frac{s_{H}}{s_{L}} \geqslant \frac{9}{8}$.

2. Now when both firms choose outputs simultaneously, we can calculate their payoffs under different cases as following.

A. The case of the public firm providing lower quality product

$$
\begin{gathered}
s_{H}=0.913549 \bar{v}, s_{L}=0.588302 \bar{v} \\
W_{L}^{S}=0.151155 \bar{v}^{3}, \pi_{H}^{S}=0.003907 \bar{v}^{3} .
\end{gathered}
$$

B. The case of the public firm providing higher quality product

$$
\begin{gathered}
s_{H}=0.731408 \bar{v}, s_{L}=0.320684 \bar{v} \\
W_{H}^{S}=0.154238 \bar{v}^{3}, \pi_{L}^{S}=0.005546 \bar{v}^{3} .
\end{gathered}
$$

It is clear that $W_{H}^{S}>W_{L}^{S}$ and $\pi_{L}^{S}>\pi_{H}^{S}$, so two firms choose quality simultaneously with the public firm acting as the higher-quality producer in the quality stage.

\subsection{Subgame perfect equilibrium}

Combining Proposition 1 and 2, we summarize the main result in the form of Theorem 1.

Theorem 1 In a quality-then-output game of endogenous timing with observable delay in a mixed duopoly with vertical differentiation, the unique subgame perfect equilibrium in pure strategies is characterized by simultaneous play in the quality stage with the public firm producing higher quality product and simultaneous play in the second period in the output stage.

The Theorem 1 reflects that there is a unique subgame perfect Nash equilibrium of the dynamic game, that is, the quality of two firms is determined endogenously in the quality stage while their moving times are given exogenously, a social welfare maximizing quality turns out to be $0.731408 \bar{v}$ for the public firm and a profit maximizing quality $0.320684 \bar{v}$ for the private firm, then they announce simultaneously that they will choice outputs in the second period, finally in the output stage the public firm will produces an output of $0.576635 \bar{v}$ while the private firm's output is $0.131512 \bar{v}$. Solving for the values of other variables one gets $W_{H}^{S}=0.154238 \bar{v}^{3}$ (social welfare of the public firm) and $\pi_{L}^{S}=0.005546 \bar{v}^{3}$ (profit of the private firm). 


\section{Comparison with the case of two private firms}

Following the same setting in the vertically differentiated mixed duopoly, we can get the equilibrium quality in the pure private duopoly by comparing the high quality and low quality firm's payoffs in Tab. 1 .

Table 1. The game in the prestage of timing choice

\begin{tabular}{cccc}
\hline & & \multicolumn{2}{c}{$L$} \\
\cline { 3 - 4 } & & \multicolumn{2}{c}{$F$} \\
\hline & $F$ & $\pi_{H}^{S}, \pi_{S}^{L}$ & $\pi_{L}^{H}, \pi_{F}^{L}$ \\
$H$ & & & \\
& $S$ & $\pi_{F}^{H}, \pi_{L}^{L}$ & $\pi_{S}^{H}, \pi_{S}^{L}$ \\
\hline
\end{tabular}

We can solve for equilibrium output in the three fixed-timing games. Once the equilibrium outputs is derived, it is then straightforward to get each firm's payoff in each fixed -timing game which is presented below.

(1) simultaneous move

$$
\begin{gathered}
\left\{s_{H}, s_{L}\right\}=\{0.738096 \bar{v}, 0.585576 \bar{v}\} \\
\pi_{H}^{S}=0.035256 \bar{v}^{3}, \pi_{L}^{S}=0.034957 \bar{v}^{3}
\end{gathered}
$$

(2) the low-quality firm acting as the output leader

$$
\begin{gathered}
\left\{s_{H}, s_{L}\right\}=\{0.844174 \bar{v}, 0.631495 \bar{v}\} \\
\pi_{H}^{F}=0.024643 \bar{v}^{3}, \pi_{L}^{L}=0.039410 \bar{v}^{3}
\end{gathered}
$$

(3) the high-quality firm acting as the output leader

$$
\begin{gathered}
\left\{s_{H}, s_{L}\right\}=\{0.706420 \bar{v}, 0.456905 \bar{v}\} \\
\pi_{H}^{L}=0.041196 \bar{v}^{3}, \pi_{L}^{F}=0.026095 \bar{v}^{3}
\end{gathered}
$$

Overall, the following chains of inequalities hold:

$$
\begin{aligned}
& \pi_{H}^{L}>\pi_{H}^{S}>\pi_{H}^{F} \\
& \pi_{L}^{L}>\pi_{L}^{S}>\pi_{L}^{F} .
\end{aligned}
$$

Using these properties, one can go back to Tab. 1 and observe that the prestage can be solved by applying iterated dominance, since (i) the high quality firm drops strategy $S$ as it is strictly dominated by $F$, (ii) the low quality firm drops what remains of strategy 
$S$, and therefore (iii) the matrix reduces to the cell $(F, F)$. This discussion allows us to formulate the Theorem 2 .

Theorem 2 The only pure strategy equilibrium in the extend output game with observable delay are simultaneous output settings since both two private firms want to choose the output leader in the output stage.

Compared with endogenous timing in the same setting in a vertical differentiated mixed duopoly, the timing of outputs is different in the output stage. We have explained why it is so in the chains of inequalities (9). Because a public firm aims at maximizing social welfare, it prefers moving in the second period in the output stage and the private firm has the same preference knowing that it would have lower profit if moving in the first period. However, this is not the case in the purely private duopoly. In the purely private duopoly, each firm prefers leadership to simultaneous play in the output stage.

Furthermore, we found that the social welfare is $0.154238 \bar{v}^{3}$ under mixed oligopolies and $0.136589 \bar{v}^{3}$ under purely private duopoly. This means that when the state-owned public firm has been privatized completely, the social welfare does not necessarily increase in vertical differentiated products markets. This also has been identified by the fact that in many developing countries, especially in communism countries, the privatization of state-owned public firm does not always make the social welfare get better.

\section{Conclusion}

In this paper, we consider the endogenous order of moves in the observable delay game of Hamiton and Slutsky [6] in the context of a "three-stage" game of quality and output setting in a mixed duopoly. We find that the unique subgame perfect equilibrium in pure strategies is characterized by simultaneous play with the public firm acting as the high quality producer in the quality stage and simultaneous play in the second period in the output stage. The result is different from the one in a purely private duopoly which is characterized by simultaneous play in the quality stage and simultaneous play in the first period in the output stage.

The subgame perfect equilibrium obtained in our analysis is the consequence of interaction between the public firm and the private firm. The public firm acting as the high quality producer wants to move in the second period in the output stage since it aims at maximizing social welfare. Anticipating the public firm's behavior, the private firm does not want to be a leader either. 


\section{Appendix A}

In the Appendix A, we show how to derive each firm's payoff in fixed-timing games when firms compete in outputs, namely, the simultaneous-name, public-leader and private-leader games. Let the superscripts ' $S$ ', ' $L$ ' and ' $F$ ' denote 'simultaneous-mover', 'leader' and 'follower', respectively.

\section{(a) The case of the public firm providing lower quality product}

In this case, we can obtain

$$
W_{L}=\bar{v} s_{H} x_{H}-\frac{1}{2} s_{H}^{2} x_{H}-\frac{1}{2} s_{H} x_{H}^{2}+\bar{v} s_{L} x_{L}-\frac{1}{2} s_{L}^{2} x_{L}-s_{L} x_{H} x_{L}-\frac{1}{2} s_{L} x_{L}^{2}
$$

and

$$
\pi_{H}=\left(s_{H}\left(\bar{v}-x_{H}\right)-s_{L} x_{L}-\frac{1}{2} s_{H}^{2}\right) x_{H} .
$$

In the simultaneous-move game, we first obtain each firm's output reaction function as the following:

$$
\begin{gathered}
x_{L}=\frac{1}{2}\left(2 \bar{v}-s_{L}-2 x_{H}\right) \\
x_{H}=\frac{2 \bar{v} s_{H}-s_{H}^{2}-2 s_{L} x_{L}}{4 s_{H}} .
\end{gathered}
$$

Once the reaction functions are obtained, one can then solve for the equilibrium outputs in the three fixed-timing games. Once the equilibrium outputs are derived, it is then straightforward to get each firm's payoff in each fixed-timing game which is presented below.

(1) Simultaneous move

$$
\begin{gathered}
x_{L}^{S}=\frac{s_{H}\left(2 \bar{v}+s_{H}-2 s_{L}\right)}{2\left(2 s_{H}-s_{L}\right)}, x_{H}^{S}=\frac{\left(s_{H}-s_{L}\right)\left(2 \bar{v}-s_{H}-s_{L}\right)}{2\left(2 s_{H}-s_{L}\right)} \\
W_{L}^{S}=\left[s _ { H } \left(12 \bar{v}^{2} s_{H}^{2}-12 \bar{v} s_{H}^{3}+3 s_{H}^{4}-12 \bar{v}^{2} s_{H} s_{L}+16 \bar{v} s_{H}^{2} s_{L}-s_{H}^{3} s_{L}+4 \bar{v}^{2} s_{L}^{2}-8 \bar{v} s_{H} s_{L}^{2}-\right.\right. \\
\left.\left.6 s_{H}^{2} s_{L}^{2}+6 s_{H} s_{L}^{3}-s_{L}^{4}\right)\right]:\left[8\left(2 s_{H}-s_{L}\right)^{2}\right] \\
\pi_{H}^{S}=\frac{s_{H}\left(s_{H}-s_{L}\right)^{2}\left(2 \bar{v}-s_{H}-s_{L}\right)^{2}}{4\left(2 s_{H}-s_{L}\right)^{2}}
\end{gathered}
$$

(2) Public leader

$$
x_{L}^{L}=\frac{s_{H}\left(2 \bar{v}+3 s_{H}-4 s_{L}\right)}{2\left(4 s_{H}-3 s_{L}\right)}, \quad x_{H}^{F}=\frac{\left(s_{H}-s_{L}\right)\left(2 \bar{v}-s_{H}-s_{L}\right)}{4 s_{H}-3 s_{L}}
$$




$$
\begin{gathered}
W_{L}^{L}=\frac{s_{H}\left(12 \bar{v}^{2} s_{H}-12 \bar{v} s_{H}^{2}+3 s_{H}^{3}-8 \bar{v}^{2} s_{L}+12 \bar{v} s_{H} s_{L}-4 \bar{v} s_{L}^{2}-6 s_{H} s_{L}^{2}+4 s_{L}^{3}\right)}{8\left(4 s_{H}-3 s_{L}\right)} \\
\pi_{H}^{F}=\frac{s_{H}\left(s_{H}-s_{L}\right)^{2}\left(2 \bar{v}-s_{H}-s_{L}\right)^{2}}{\left(4 s_{H}-3 s_{L}\right)^{2}}
\end{gathered}
$$

(3) Public follower

$$
\begin{gathered}
x_{L}^{F}=\frac{1}{4}\left(2 \bar{v}+s_{H}-s_{L}\right), \quad x_{H}^{L}=\frac{1}{4}\left(2 \bar{v}-s_{H}-s_{L}\right) \\
W_{L}^{F}=\frac{1}{32}\left(12 \bar{v}^{2} s_{H}-12 \bar{v} s_{H}^{2}+3 s_{H}^{3}+4 \bar{v}^{2} s_{L}+3 s_{H}^{2} s_{L}-4 \bar{v} s_{L}^{2}-3 s_{H} s_{L}^{2}+s_{L}^{3}\right) \\
\pi_{H}^{L}=\frac{1}{16}\left(s_{H}-s_{L}\right)\left(2 \bar{v}-s_{H}-s_{L}\right)^{2}
\end{gathered}
$$

Clearly, for the public firm's payoff we obtain the following results:

$$
\begin{gathered}
W_{L}^{S}-W_{L}^{L}=-\frac{s_{H} s_{L}\left(s_{H}-s_{L}\right)^{2}\left(2 \bar{v}-s_{H}-s_{L}\right)^{2}}{8\left(4 s_{H}-3 s_{L}\right)\left(2 s_{H}-s_{L}\right)^{2}}<0 \\
W_{L}^{S}-W_{L}^{F}=-\frac{s_{L}\left(s_{H}-s_{L}\right)\left(4 s_{H}-s_{L}\right)\left(2 \bar{v}-s_{H}-s_{L}\right)^{2}}{32\left(2 s_{H}-s_{L}\right)^{2}}<0 \\
W_{L}^{L}-W_{L}^{F}=-\frac{3 s_{L}\left(s_{H}-s_{L}\right)\left(2 \bar{v}-s_{H}-s_{L}\right)^{2}}{32\left(4 s_{H}-3 s_{L}\right)}<0
\end{gathered}
$$

So, we have $W_{L}^{F}>W_{L}^{L}>W_{L}^{S}$.

For the private firm's payoff, we can find

$$
\begin{aligned}
& \pi_{H}^{S}-\pi_{H}^{F}=-\frac{s_{H} s_{L}\left(8 s_{H}-5 s_{L}\right)\left(s_{H}-s_{L}\right)^{2}\left(2 \bar{v}-s_{H}-s_{L}\right)^{2}}{4\left(4 s_{H}-3 s_{L}\right)^{2}\left(2 s_{H}-s_{L}\right)^{2}}<0 \\
& \pi_{H}^{S}-\pi_{H}^{L}=-\frac{s_{L}^{2}\left(s_{H}-s_{L}\right)\left(2 \bar{v}-s_{H}-s_{L}\right)^{2}}{16\left(2 s_{H}-s_{L}\right)^{2}}<0 \\
& \pi_{H}^{F}-\pi_{H}^{L}=\frac{s_{L}\left(s_{H}-s_{L}\right)\left(8 s_{H}-9 s_{L}\right)\left(2 \bar{v}-s_{H}-s_{L}\right)^{2}}{16\left(4 s_{H}-3 s_{L}\right)^{2}} \begin{cases}<0 & \text { if } 1<\frac{s_{H}}{s_{L}}<\frac{9}{8} \\
\geqslant 0 & \text { if } \frac{s_{H}}{s_{L}} \geqslant \frac{9}{8}\end{cases}
\end{aligned}
$$

Summarizing the inequalities above, the following chains of inequalities hold:

$$
\begin{aligned}
& \pi_{H}^{L}>\pi_{H}^{F}>\pi_{H}^{S} \quad \text { if } 1<\frac{s_{H}}{s_{L}}<\frac{9}{8} \\
& \pi_{H}^{F}>\pi_{H}^{L}>\pi_{H}^{S} \quad \text { if } \quad \frac{s_{H}}{s_{L}} \geqslant \frac{9}{8} .
\end{aligned}
$$




\section{(b) The case of the public firm providing higher quality product}

In this case, following the same procedure in section (a), we can also get each firm's payoff in each fixed-timing game

$$
\begin{gathered}
W_{H}=\bar{v} s_{H} x_{H}-\frac{1}{2} s_{H}^{2} x_{H}-\frac{1}{2} s_{H} x_{H}^{2}+\bar{v} s_{L} x_{L}-\frac{1}{2} s_{L}^{2} x_{L}-s_{L} x_{H} x_{L}-\frac{1}{2} s_{L} x_{L}^{2} \\
\pi_{L}=\left(\left(\bar{v}-x_{H}-x_{L}\right) s_{L}-\frac{1}{2} s_{L}^{2}\right) x_{L} .
\end{gathered}
$$

In the simultaneous-move game, we first obtain each firm's output reaction function as the following:

$$
\begin{aligned}
x_{H} & =\frac{2 \bar{v} s_{H}-s_{H}^{2}-2 s_{L} x_{L}}{2 s_{H}} \\
x_{L} & =\frac{1}{4}\left(2 \bar{v}-s_{L}-2 x_{H}\right) .
\end{aligned}
$$

(1) Simultaneous move

$$
\begin{gathered}
x_{H}^{S}=-\frac{-4 \bar{v} s_{H}+2 s_{H}^{2}+2 \bar{v} s_{L}-s_{L}^{2}}{2\left(2 s_{H}-s_{L}\right)}, x_{L}^{S}=\frac{s_{H}\left(s_{H}-s_{L}\right)}{2\left(2 s_{H}-s_{L}\right)} \\
W_{H}^{S}=\left[s _ { H } \left(16 \bar{v}^{2} s_{H}^{2}-16 \bar{v} s_{H}^{3}+4 s_{H}^{4}-16 \bar{v}^{2} s_{H} s_{L}+16 \bar{v} s_{H}^{2} s_{L}-s_{H}^{3} s_{L}+4 \bar{v}^{2} s_{L}^{2}-4 \bar{v} s_{H} s_{L}^{2}-\right.\right. \\
\left.\left.6 s_{H}^{2} s_{L}^{2}+5 s_{H} s_{L}^{3}-s_{L}^{4}\right)\right]:\left[8\left(2 s_{H}-s_{L}\right)^{2}\right] \\
\pi_{L}^{S}=\frac{s_{H}^{2}\left(s_{H}-s_{L}\right)^{2} s_{L}}{4\left(2 s_{H}-s_{L}\right)^{2}}
\end{gathered}
$$

(2) Public leader

$$
\begin{gathered}
x_{H}^{L}=\frac{8 \bar{v} s_{H}-4 s_{H}^{2}-6 \bar{v} s_{L}+3 s_{L}^{2}}{2\left(4 s_{H}-3 s_{L}\right)}, \quad x_{L}^{F}=\frac{s_{H}\left(s_{H}-s_{L}\right)}{4 s_{H}-3 s_{L}} \\
W_{H}^{F}=\frac{s_{H}}{32}\left(16 \bar{v}^{2}-16 \bar{v} s_{H}+4 s_{H}^{2}+3 s_{H} s_{L}-3 s_{L}^{2}\right) \\
\pi_{L}^{L}=\frac{s_{H} s_{L}}{16}\left(s_{H}-s_{L}\right)
\end{gathered}
$$

Clearly, for the public firm's payoff we can obtain

$$
\begin{aligned}
& W_{H}^{S}-W_{H}^{L}=-\frac{s_{H}^{2} s_{L}^{2}\left(s_{H}-s_{L}\right)^{2}}{8\left(4 s_{H}-3 s_{L}\right)\left(2 s_{H}-s_{L}\right)^{2}}<0 \\
& W_{H}^{S}-W_{H}^{F}=-\frac{s_{H} s_{L}^{2}\left(s_{H}-s_{L}\right)\left(4 s_{H}-s_{L}\right)}{32\left(2 s_{H}-s_{L}\right)^{2}}<0
\end{aligned}
$$




$$
W_{H}^{L}-W_{H}^{F}=-\frac{3 s_{H} s_{L}^{2}\left(s_{H}-s_{L}\right)}{32\left(4 s_{H}-3 s_{L}\right)}<0
$$

So, we have $W_{H}^{F}>W_{H}^{L}>W_{H}^{S}$.

For the private firm's payoff, we can find

$$
\begin{gathered}
\pi_{L}^{S}-\pi_{L}^{F}=-\frac{s_{H}^{2} s_{L}^{2}\left(8 s_{H}-5 s_{L}\right)\left(s_{H}-s_{L}\right)^{2}}{4\left(4 s_{H}-3 s_{L}\right)^{2}\left(2 s_{H}-s_{L}\right)^{2}}<0 \\
\pi_{L}^{S}-\pi_{L}^{L}=-\frac{s_{H} s_{L}^{3}\left(s_{H}-s_{L}\right)}{16\left(2 s_{H}-s_{L}\right)^{2}}<0 \\
\pi_{L}^{F}-\pi_{L}^{L}=\frac{s_{H} s_{L}^{2}\left(s_{H}-s_{L}\right)\left(8 s_{H}-9 s_{L}\right)}{16\left(4 s_{H}-3 s_{L}\right)^{2}}\left\{\begin{array}{l}
<0 \quad \text { if } 1<\frac{s_{H}}{s_{L}}<\frac{9}{8} \\
\geqslant 0 \quad \text { if } \frac{s_{H}}{s_{L}} \geqslant \frac{9}{8} .
\end{array}\right.
\end{gathered}
$$

Summarizing the inequalities above, the following chains of inequalities hold:

$$
\begin{aligned}
& \pi_{H}^{L}>\pi_{H}^{F}>\pi_{H}^{S} \quad \text { if } 1<\frac{s_{H}}{s_{L}}<\frac{9}{8} \\
& \pi_{H}^{F}>\pi_{H}^{L}>\pi_{H}^{S} \quad \text { if } \frac{s_{H}}{s_{L}} \geqslant \frac{9}{8} .
\end{aligned}
$$

\section{Appendix B}

(a) Assuming that the differentiation of equilibrium quality of products between the public and private firm is less than $\frac{9}{8}$.

(1) The public firm acting as an output follower with low quality.

In this case, the social welfare for the public firm is given by

$$
W_{L}^{F}=\int_{\bar{v}-x_{H}}^{\bar{v}} \theta s_{H} \mathrm{~d} \theta+\int_{\bar{v}-x_{H}-x_{L}}^{\bar{v}-x_{H}} \theta s_{L} \mathrm{~d} \theta-\frac{1}{2} s_{H}^{2} x_{H}-\frac{1}{2} s_{L}^{2} x_{L} .
$$

Maximizing $W_{L}^{F}$ with respect to $x_{L}$, one gets the best output reaction function of the public firm as $x_{L}=\frac{1}{2}\left(2 \bar{v}-s_{L}-2 x_{H}\right)$.

Looking at the profit function of the private firm and substituting in the public firm's best output action function (in keeping with the notion of backward induction), one gets $\pi_{H}^{L}=\frac{1}{2}\left(s_{H}-s_{L}\right) x_{H}\left(2 \bar{v}-s_{H}-s_{L}-2 x_{H}\right)$. Maximizing $\pi_{H}^{L}$, with respect to $x_{H}$, and solving for $x_{H}$, one gets $x_{H}^{L}=\frac{1}{4}\left(2 \bar{v}-s_{H}-s_{L}\right)$ and $x_{L}^{F}=\frac{1}{4}\left(2 \bar{v}+s_{H}-s_{L}\right)$. 
After simple albeit tedious substitutions, the objective functions of the public and private firm simplify as

$$
\begin{gathered}
W_{L}^{F}=\frac{1}{32}\left(12 \bar{v}^{2} s_{H}-12 \bar{v} s_{H}^{2}+3 s_{H}^{3}+4 \bar{v}^{2} s_{L}+3 s_{H}^{2} s_{L}-4 \bar{v} s_{L}^{2}-3 s_{H} s_{L}^{2}+s_{L}^{3}\right) \\
\pi_{H}^{L}=\frac{1}{16}\left(s_{H}-s_{L}\right)\left(2 \bar{v}-s_{H}-s_{L}\right)^{2} .
\end{gathered}
$$

Differentiating (19) and (20) with respect to $s_{L}$ and $s_{H}$, we derive the value $s_{H}=0.845299 \bar{v}$ and $s_{L}=0.535898 \bar{v}$, which mean that the differentiation of equilibrium quality of products between the public and private firm, that is, $\frac{s_{H}}{s_{L}}=1.577350$, contradict with the assumption $\frac{s_{H}}{s_{L}}<\frac{9}{8}$.

(2) The public firm acting as an output follower with high quality.

In this case, following the same procedure as (1) in section (a), we get the equilibrium quality $s_{H}=0.751474 \bar{v}$ and $s_{L}=0.375737 \bar{v}$, which also signify that the differentiation of equilibrium quality of products between the public and private firm, that is, $\frac{s_{H}}{s_{L}}=2$, contradict with the assumption $\frac{s_{H}}{s_{L}}<\frac{9}{8}$.

\section{(b) Assuming that the differentiation of equilibrium quality of products between the public and private firm is not less than $\frac{9}{8}$.}

(1) Simultaneous play in the output stage with the public firm producing low quality product.

The best output action functions of the public and private firm are given respectively by $x_{L}=\frac{1}{2}\left(2 \bar{v}-s_{L}-2 x_{H}\right)$ and $x_{H}=\frac{2 \bar{v}_{H}-s_{H}^{2}-2 s_{L} x_{L}}{4 s_{H}}$. Maximizing firms' objective function with respect to their own output, we can get the optimal outputs produced by the public and private firm as

$$
\begin{aligned}
& x_{L}^{S}=\frac{s_{H}\left(2 \bar{v}+s_{H}-2 s_{L}\right)}{2\left(2 s_{H}-s_{L}\right)} \\
& x_{H}^{S}=\frac{\left(s_{H}-s_{L}\right)\left(2 \bar{v}-s_{H}-s_{L}\right)}{2\left(2 s_{H}-s_{L}\right)}
\end{aligned}
$$

Then, their corresponding objective functions are given by

$$
\begin{gathered}
W_{L}^{S}=\left[s _ { H } \left(12 \bar{v}^{2} s_{H}^{2}-12 \bar{v} s_{H}^{3}+3 s_{H}^{4}-12 \bar{v}^{2} s_{H} s_{L}+16 \bar{v} s_{H}^{2} s_{L}-s_{H}^{3} s_{L}+4 \bar{v}^{2} s_{L}^{2}-8 \bar{v} s_{H} s_{L}^{2}-\right.\right. \\
\left.\left.6 s_{H}^{2} s_{L}^{2}+6 s_{H} s_{L}^{3}-s_{L}^{4}\right)\right]:\left[8\left(2 s_{H}-s_{L}\right)^{2}\right] \\
\pi_{H}^{S}=\frac{4 \bar{v}^{2} s_{H}^{3}-4 \bar{v} s_{H}^{4}+s_{H}^{5}-8 \bar{v}^{2} s_{H}^{2} s_{L}+4 \bar{v} s_{H}^{3} s_{L}+4 \bar{v}^{2} s_{H} s_{L}^{2}+4 \bar{v} s_{H}^{2} s_{L}^{2}-2 s_{H}^{3} s_{L}^{2}-4 \bar{v} s_{H} s_{L}^{3}+s_{H} s_{L}^{4}}{4\left(2 s_{H}-s_{L}\right)^{2}}
\end{gathered}
$$


We now look for the solutions of the quality game. Firms will choose their quality specifications to maximize their objective functions. The first conditions of this problem are:

$$
\begin{aligned}
& \frac{\partial W_{L}^{S}}{\partial s_{L}}=\frac{s_{H}\left(8 \bar{v} s_{H}^{3}+4 s_{H}^{4}+4 \bar{v}^{2} s_{H} s_{L}-16 \bar{v} s_{H}^{2} s_{L}-25 s_{H}^{3} s_{L}+36 s_{H}^{2} s_{L}^{2}-14 s_{H} s_{L}^{3}+2 s_{L}^{4}\right)}{8\left(2 s_{H}-s_{L}\right)^{3}}=0 \\
& \frac{d \pi_{H}^{S}}{d s_{H}}=\frac{\left(s_{H}-s_{L}\right)\left(-2 \bar{v}+s_{H}+s_{L}\right)\left(-4 \bar{v} s_{H}^{2}+6 s_{H}^{3}+2 \bar{v} s_{H} s_{L}-5 s_{H}^{2} s_{L}-2 \bar{v} s_{L}^{2}+2 s_{H} s_{L}^{2}+s_{L}^{3}\right)}{4\left(2 s_{H}-s_{L}\right)^{3}}=0
\end{aligned}
$$

We can get the equilibrium quality as $s_{H}=0.913549 \bar{v}$ and $s_{L}=0.588302 \bar{v}$, which mean that the differentiation of equilibrium quality of products between the public and private firm, that is, $\frac{s_{H}}{s_{L}}=1.552857$, satisfies the assumption $\frac{s_{H}}{s_{L}} \geqslant \frac{9}{8}$.

(2) Simultaneous play in the output stage with the public firm producing high quality product.

In this case, following the same procedure as (1) in section (b), we can obtain the equilibrium quality $s_{H}=0.731408 \bar{v}$ and $s_{L}=0.320684 \bar{v}$, which also signify that the differentiation of equilibrium quality of products between the public and private firm, that is, $\frac{s_{H}}{s_{L}}=2.280776$, satisfies the assumption $\frac{s_{H}}{s_{L}} \geqslant \frac{9}{8}$.

\section{References}

[1] J. BA'RCENA-RUIZ: Endogenous timing in a mixed duopoly: price competition. J. Economics, 91(3), (2007), 263-72.

[2] J.C. CHOI and H.S. SHIN: A comment on a model of vertical product differentiation. J. Industrial Economics, 60(2), (1992), 229-231.

[3] H. Cremer, M. Marchand and J.F. Thisse: Mixed oligopoly with differentiated products. Int. J. Industrial Organization, 9(1), (1991), 43-53.

[4] F. Delbono, V. Denicolo and C. Scarpa: Quality choice in a vertically differentiated mixed duopoly. Economics Notes, 25(1), (1996), 33-46.

[5] I. GRILO: Mixed Duopoly under Vertical Differentiation. Annales d'Économie et de Statistique, 33 (1994), 92-112.

[6] J. HAmilton and S. SLUTSKY: Endogenous timing in duopoly games: Stackelberg or Cournot equilibria. Games and Economic Behavior, 2(1), (1990), 29-46.

[7] K. Ishibashi and T.KaneKo: Partial privatization in mixed duopoly with price and quality competition. J. Economics, 95(3), (2008), 213-31. 
[8] L. LIU and Y. LU: Endogenous timing in a mixed duopoly with endogenous vertical differentiation. Bulletin of Economic Research, 66(3), (2012), 305-312.

[9] Y. LU: Endogenous timing in a mixed oligopoly with foreign competitors: the linear demand case. J. Economics, 88(1), (2006), 49-68.

[10] S. LuTZ and M. PEZZINO: Vertically differentiated mixed oligopoly with qualitydependent fixed costs. Manchester School, 82(5), (2014), 596-619.

[11] M. MотTA: Endogenous quality choice: price vs. quantity competition. J. Industrial Economics, 41(2), (1993), 113-131.

[12] D. PAL: Endogenous timing in a mixed oligopoly. Economics Letters, 61(2), (1998), 181-185.

[13] J. TIROLE: The theory of industrial organization. MIT Press, Cambridge, 1988. 